International Journal of Engineering \&Technology, $7(3.12)(2018) 656-660$
International Journal of Engineering \& Technology
SPC
Website: $w$ ww.sciencepubco.com/index.php/IJET
Research paper

\title{
Energy Analytics for Smart Meter Data using Consumer Centric Approach
}

\author{
Nimala.K ${ }^{1}$, ThamizhArasan. $\mathbf{R}^{2}$ \\ ${ }^{I}$ Department of Information Technology, SRM University, India, \\ ${ }^{2}$ Department of Computer science, Bharath University, India,

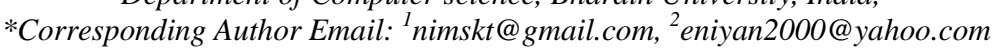

\begin{abstract}
A short-range residential consumer's demand forecasting at the distinct and cumulative level, by an analysis of data using consumer based centric approach. Energy intake behavior might fluctuate among various seasonal factors; the consumed current will change from one season to other. So hereby we are building a model which helps to calculate future electricity consumption data from the obtain ability of past smart meter data. Currently utility companies accumulate the data, use it, share for further practice, and abandon usage data at their discretion, with no input from customers. In many cases, consumers do not even have entree to their own data. But in this project Consumer can have fast admittance and control over their individual data, and also helps to choose the familiar algorithms for the data analyze rather than including third party applications. By end of analyze technique, the analyzed output will be driven to some user interactive application by creating a Graphical User Interface.
\end{abstract}

Keywords: Cluster based forecast, Data analyze, Feed forward, Graphical user interface, Smart meter.

\section{Introduction}

A smart meter helps utility companies for monitoring and billing [1], it takes a new kind of gas and electricity meter readings that can be digitally sent to energy supplier that records consumed electricity energy. It sends information in intervals of an hour or less and communicates at least on daily basis. So this can be used to estimate energy bills on the daily or monthly basis. Unlike home energy monitors, smart meters can gather data for far away reporting. The Smart Meter is an electronic device measuring natural gas or water consumption and it often refers to an energy meter. It can easily identify how much energy consumed by the meters which comes with displays. Some other smart meters, ordinarily referred to time-of-use meters or as interval [2], "Smart Meters" usually involve nearby real-time sensors, power quality monitoring and power outage notification. Such an advanced metering infrastructure [3] differs from traditional meter reading in the way that it assists two-way communications with the meter. The consumer centric approach [1] computes the energy in the following basis.

- Given the data, the consumer's choice analytic algorithm works on the given input which preserves the privacy of the data [1].

Provides storage and perseverance for the consumer's data which in turn gains full control over the data [1].

- Focuses on single view of the collected data so that analytics can be performed.

There are many techniques for prediction of energy consumption using statistical and machine learning such as Linear Regression,
ARIMA [2],[4], Generalized Additive Models, Neural Networks and Support Vector machine.

However, for very large space scales these techniques have been typically used, so as to predict the electrical load, to create data segment serving thousands of customers or even an entire country. In this paper, we focus on forecasting electricity consumption of residential [3],[5] customers, leveraging smart meter data.

\subsection{Basic Architecture}

Two vay communications

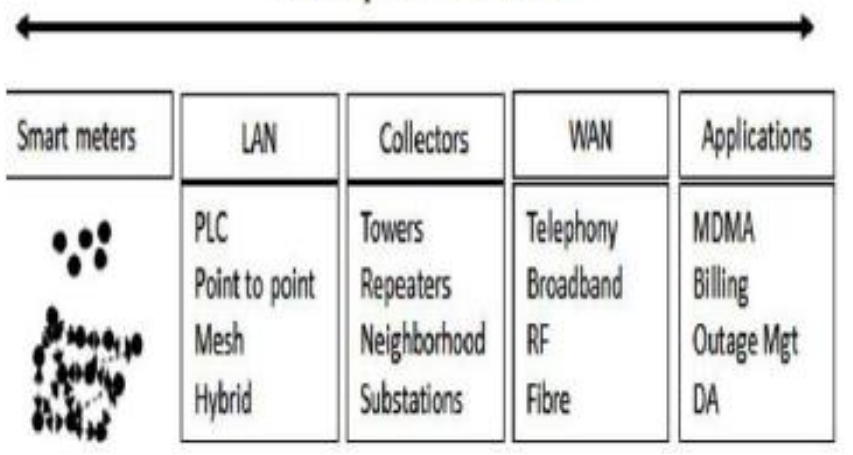

Fig. 1: Smart meter model

To reach a data crew point, smart meter collects data and transfers the reading to a local area network [6]. Energy consumption or data gives you the electricity usage which measures in kilowatt hours.

The data are collected in a frequent interval, which may vary in 15 minutes to 1 hour time gap, depending on the particular deployment and region[1, 6]. In terms of processing the data, the 
energy data are transferred to the utilities company centers by a wide area network (Fig. 1) and in some other cases data processing could be carried out at the local collection points even.

The collected data is useful for the business purposes such as billing [6], prediction, watching, summarizing and scheduling. If suppose many different electrical devices are utilized, the framework will clearly identify the consumption of the electrical gadgets. It can even tell what channel or programme is being telecasted on television and its usage of electrical consumption and the electrical noises it releases.

\subsection{Data Collection and Storage by Smart Meter}

Just alike analog meters, smart meters gather the usage of electricity against the consumer names and addresses and these information gets stored in the meters which is transmitted across the network.. Smart meter collects a data over a day and contains more information. Some utility companies receive snapshot of consumer's energy consumption through smart meters for every 15 minutes[1], while others may collect energy information on hourly basis. It is important to have strong privacy protections in place because smart meters gather the information and transfer much more data about electricity consumption.

\section{Related Work}

The utilities are looking to match the consumption of generated data for market-driven and deregulation purpose. An outdated meter only measure gas and electrical consumption, and does not provide any information about the time in which the energy was consumed at any metered location. Data procurement, transmission, processing, and interpretation done by smart meter and data analytics can be done, which bring aids to all stakeholders. To attain the above goal the stakeholder wants smart-meter to be designed and established in a way which helps them to deploy analytics for their own applications [6].According to some metric the groups of time series are discovered through kernel spectral clustering (KSC). For each cluster periodic autoregressive models are trained and then projections per cluster [7] are computed. On other hand Open TSDB, Hbase and Hadoop are Open source tools that helps to perform analytics on the time series data and store these data to get power consumption insights. As we know consumers do not have access to their data in many conditions. So to provide fast control and access over their custom data we address by designing and implementing a consumer-based centric approach which helps the consumer to analyze their data[8] based on their own applications that analyze data in a secret way where no one can access it.

The person involving in recording the units achieve different rates to be priced for energy which eliminates the cost of a monthly visit and time of day. The real time usage and rates could be informed to the users by the most advanced design .For prediction of power consumption, statistical and machine learning algorithms [6] are used. In other case we are building an GUI based integration which will directly analyze and present the outcome of the analyzed data.

\section{Learning Algorithms}

Various learning algorithms such as supervised learning, unsupervised learning and reinforcement learning have been used to forecast large-scale electricity demand. Through these learning paradigms, the literature suggests some supervised learning methods. Among those are Support vector regression (SVR), one of the most effective model to forecast future energy consumption. In this work, artificial neural network a machine learning algorithm is used for analyzing smart meter data. Meanwhile we are training a neural network model by feeding data into it, Which will help to minimize the cost by predicting future consumed electricity data.

Learning Algorithm: There are many algorithms which will train the network based upon some parameters values to the available fixed data set. To train the network the data should undergo some significant amount of practices.

Model selection: Model selection helps to represent data and applications in a significant way and also it can get rid of few challenges, faced in choosing different model.

Sturdiness (robust): The final outcome of artificial neural network will be robust based on the algorithm and cost functions chosen.

\section{Implementation Analysis}

\subsection{The Dred (Dutch Residential Energy Dataset)}

The DRED smart meter data collected from a household in the Netherland through the R\&D department. The dataset [9] contains an appliance level devices energy consumption dataset. For our proposed work we are focusing on the appliance level data for the examination. The collected data helps to check the concert of energy, invent and analyze demand retort algorithms and behavior of appliance usages.

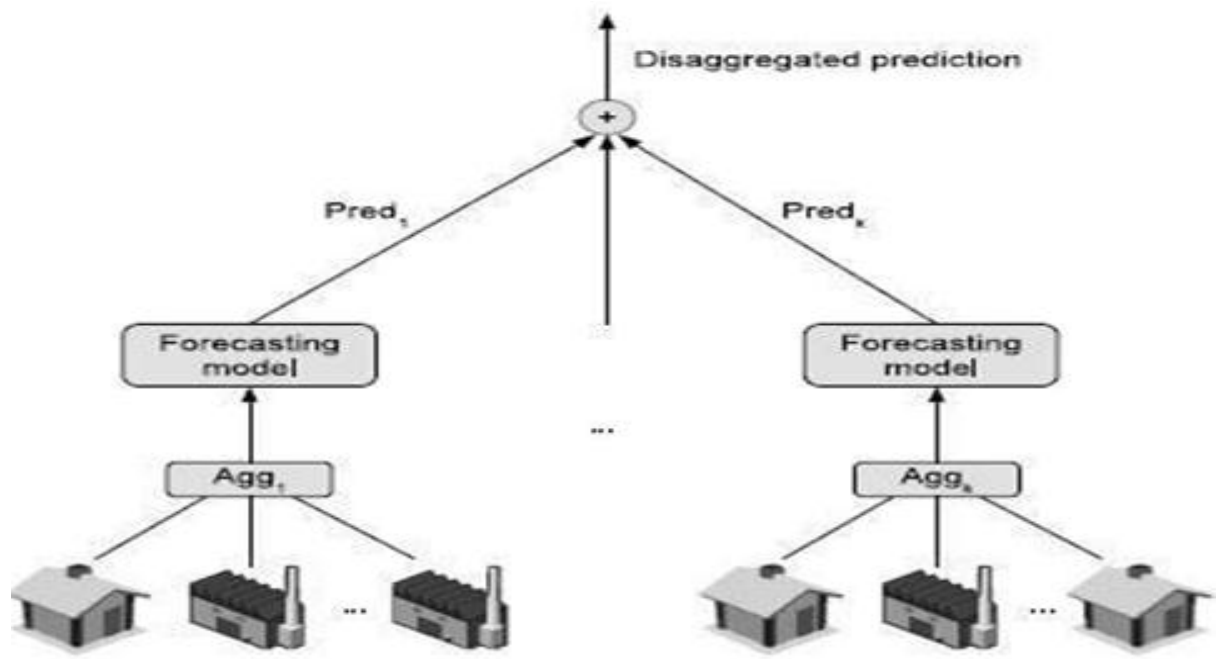

Fig. 2: Data Collection and Fragmentation 


\subsection{Appliance Level}

We used smart plug-ins to collect appliance level energy consumption data. There are 12 smart plugs were installed to monitor the appliances across the household. The plug-ins installed in the household communicates via Zigbee protocol by forming a mesh network and finally the data will be stored for the billing purpose.

In the household appliance level, some machines have multiple states where others have only two states of operation. Smart meter capture the data at regular intervals and it transfer the same data in a high speed transfer rates. Since the smart meter is enabled with new technological abilities there is a competition among the utilities. The collected data are dissimilar in the series which will be used to forecast (Fig. 4) [10] and for some variability issues.

For this work we are trying to develop an MATLAB based GUI (also known as graphical user interfaces) which helps to provide a single click point to control the applications, and it removes the need of learning language or to type commands in edit to run the application.

The GUI typically has gearshifts such as menu, toolbar, button, and slider. App Designer is environment for building apps. The applications [11] are independent programs with GUI that automate the task and design. GUI integrates some primary tasks of application building, laying out the visual components and allows to quickly move between graphic design in the canvas and code development.

We just compared our proposed system with the existing system which used to analyze and store the data. Google power meter Hohm is a commercial software solutions, being used for analytics on a fixed set of data. we can check on data privacy as well .It provides data in a different way with a slight change in values but the only drawback is that it burdens them with data maintenance through tools, which helps for run integrity check. The smart meter dataset used for analysis is examined, (Fig. 2) and fragmented into different parts depending upon the aspects present in to it and analyze is carried out.[12].The consumers can choose their familiar algorithm for the data to perform analyze. An overview of autoregressive moving average (ARMA) model is ARIMA model which is an autoregressive integrated moving average which helps to evaluate time series data. Both models are fixed to time series data either to forecast future points in the series or to better know the data. ARIMA [13-14] models are useful in some circumstances where data show sign of nonstationary. The analyzed data are shown as graphical representation in Figure 3 a) and b)

\subsection{K-Means C1ustering}

$\mathrm{K}$-means is a unsupervised learning algorithm helps to overcome the cluster problems. This simple algorithm partitions data to form a group of cluster by taking a centroid point among the each group, The Fig. 3 shows the formation of cluster with and without adjustments.

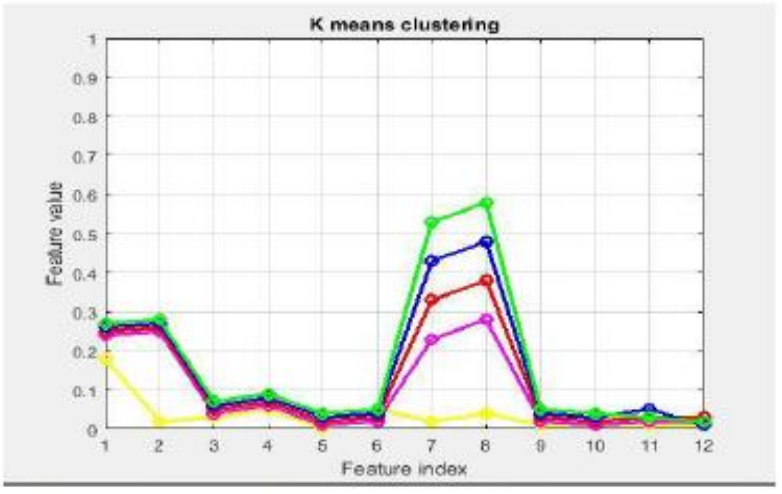

(a)

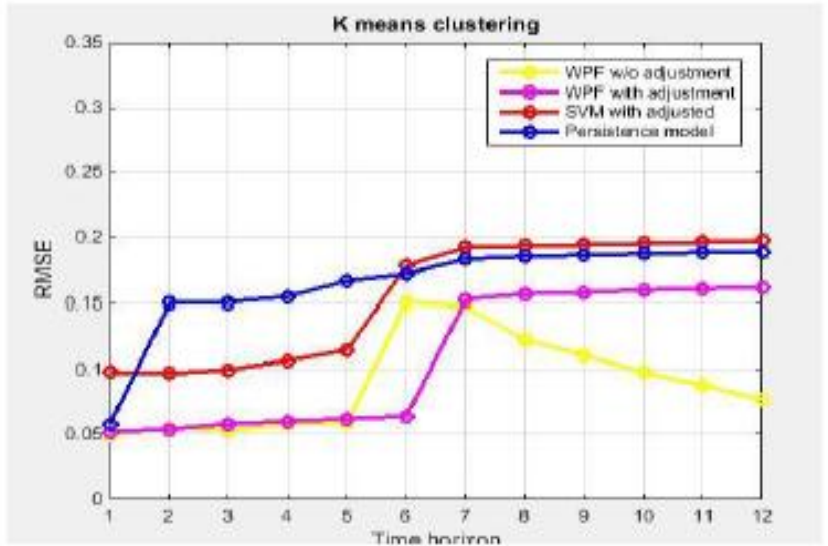

(b)

Fig. 3: K-Means: (a) K-Means clustering (b) With and without adjustments

\subsection{ARIMA Model}

Autoregressive Integrated Moving Average which helps to evaluate time series data. The time series data are actually classified into two types based upon the values present into it. (ie) Stationary data and Non Stationary data.

- Stationary data contains the value which will not change or fluctuate over the time, this means there won't be a growth and it remains horizontally along the time series. Importantly the mean and variance does not change at any cause.

- In opposite, non-stationary data will change and fluctuate among the mean and variance.

In this work model a forecast is done on our data. Forecasting helps to estimate the future values with the available past values for the time series data. In other hand prediction helps to estimate current, past, future value through regression on the data.

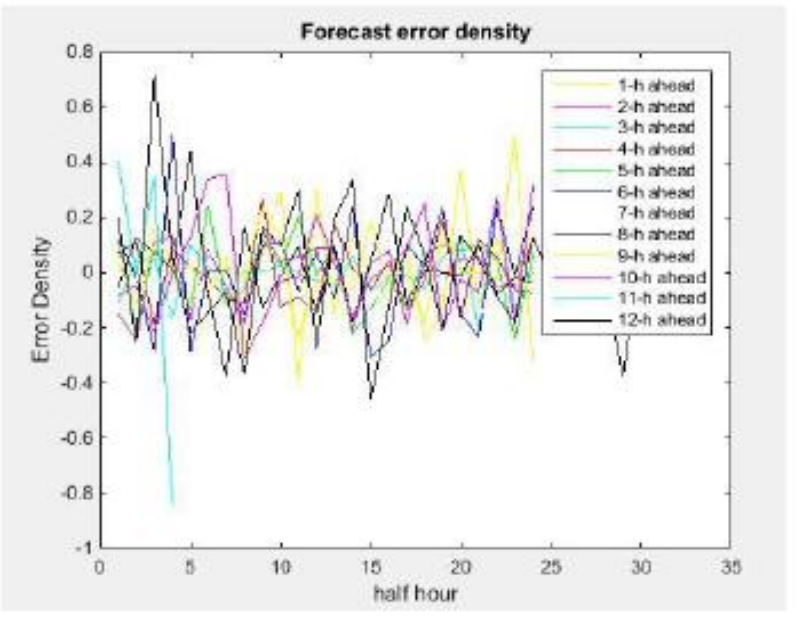

Fig. 4: Forecast Error Density

Using ARIMA model, the forecast analyzer result is shown in Fig.4.The forecast error density means the difference between the actual and predicted value for the time series data. In this figure the error density is calculated for each and every hour with the given smart meter data 


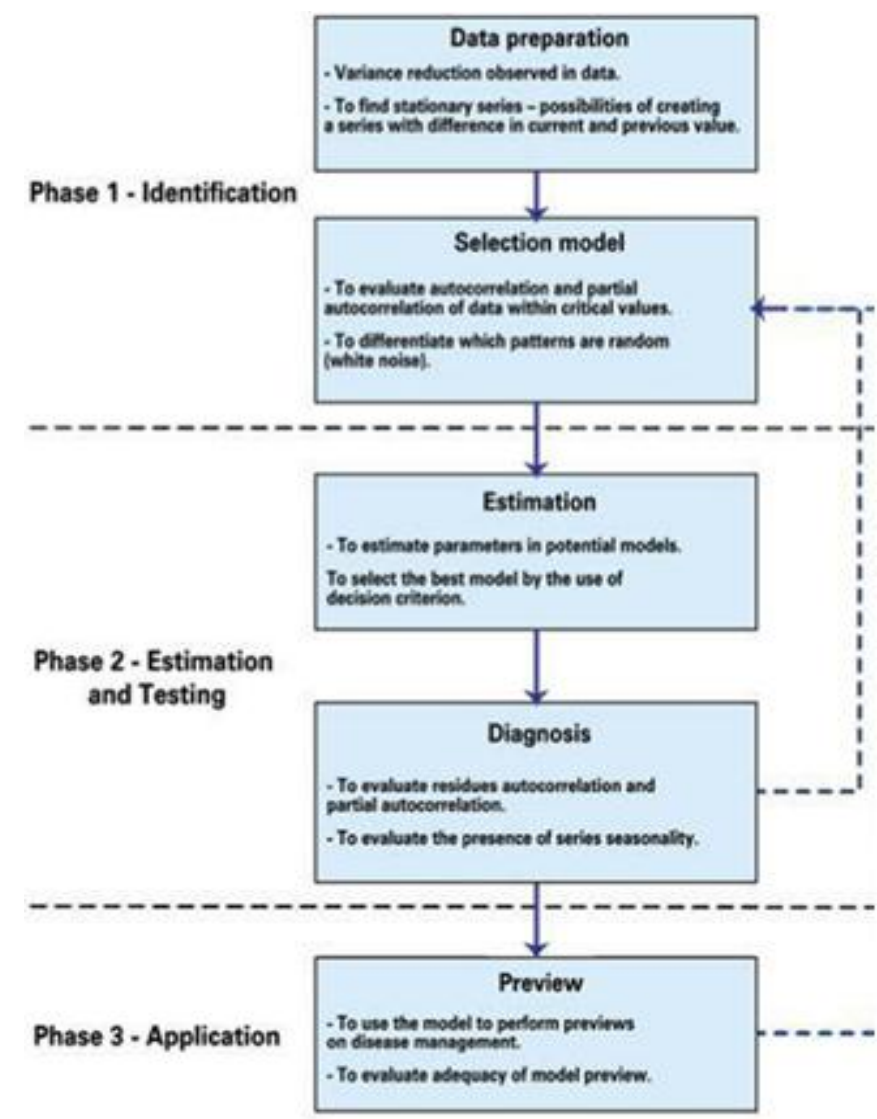

Fig. 5: Scheme for the use of Box-Jenkins Methodology (ARIMA Model)

\subsection{Feed Forward Method}

Feedforward is a network which contains $\mathrm{N}$ series number of layers. The network input is given to the first layer of the connection. Every other following layer is connecting with the previous layer as shown in the Fig.5. The output is derived from the final or the last layer in the network. This method is mainly used for the input output mapping [15]. Any input output mapping problems in a network can be solved by one hidden layer which contains enough neurons present in feed forward network.

Feedforward is an artificial neural network, which as connections but it does not form a cycle, ie it is not a regular way of forming a network connection. This network moves an information in one way direction (i.e.) from the input node to output node through the hidden node in between. So this will resolve the problem related loop or cycle formation.

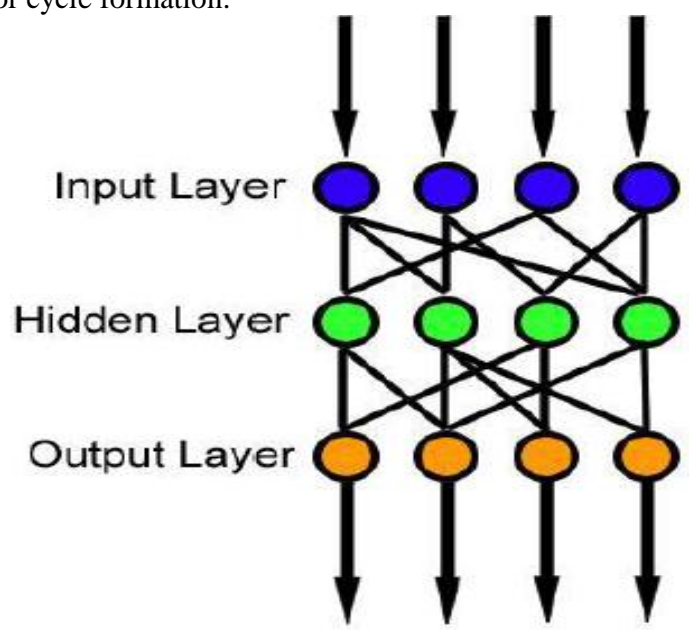

Fig. 6: Feedforward neural network

In this work, the classification algorithm is used to train the nodes based upon the previous node value using the neuron like processing units, mean while it helps to predict the data among the set of datasets. The below is the prediction obtained with the smart meter data Fig.7.

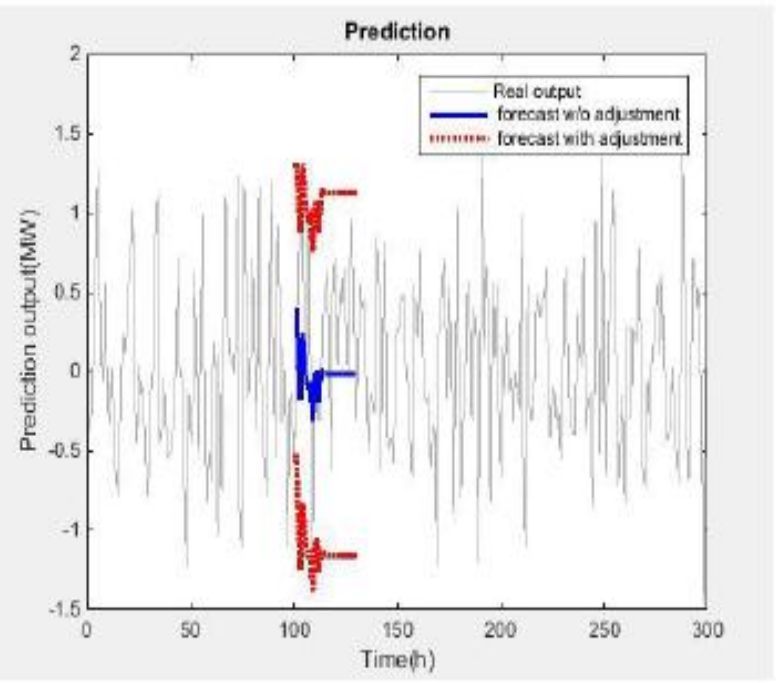

Fig. 7: Prediction

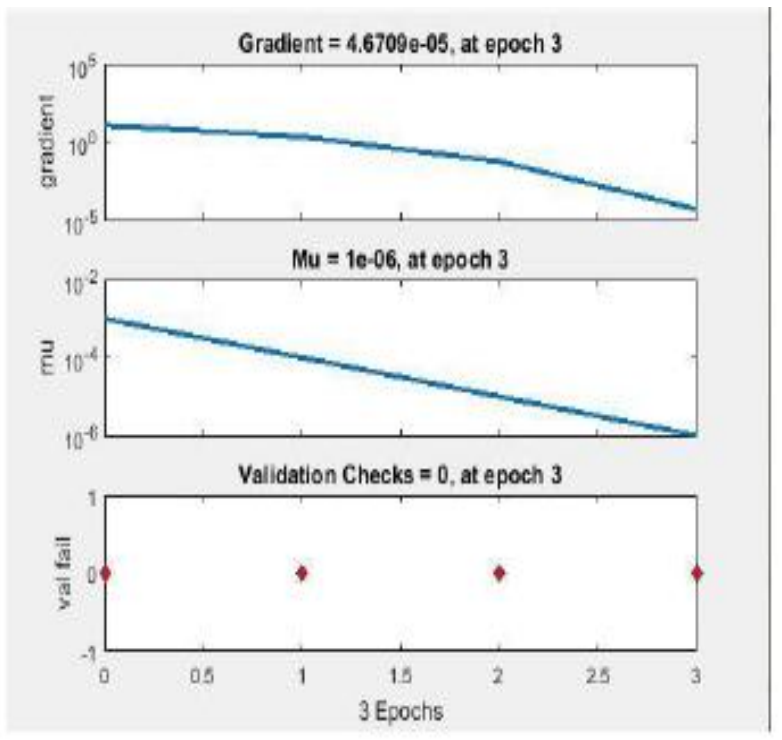

Fig. 7: Training state

\subsection{Regression}

The regression [16] helps to study the relationship between two continuous variable for the given smart meter data. The visual representation for the regression analysis is shown which indicates the straight line which means the data is fit and trained for examining.

Linear regression is a model which is used to build a relationship between dependent and explanatory variable.

- Dependent variable is one which depends upon other variable, so it does get affected easily.

- Explanatory variable is an independent variable, so it does not get affected with the any other variable. 


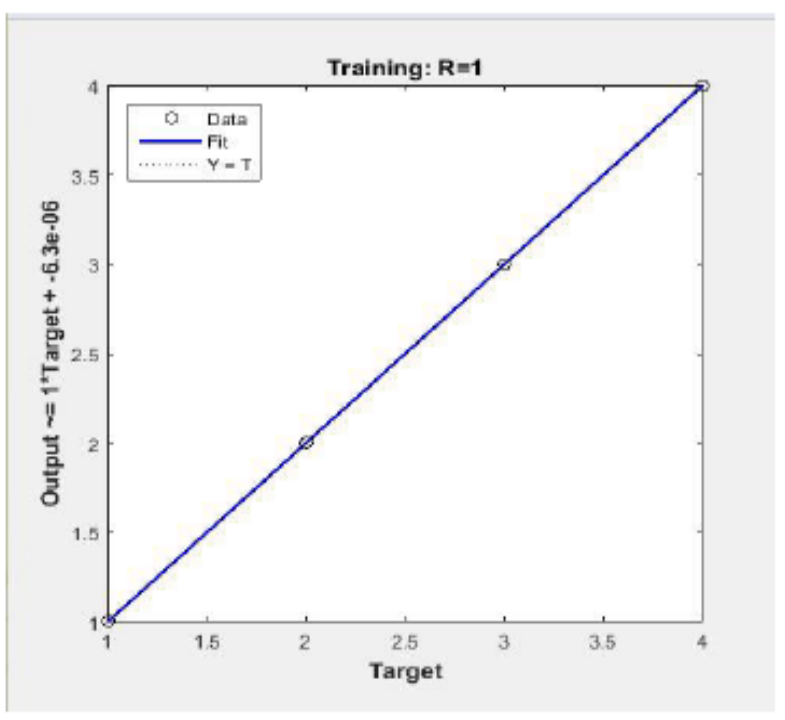

Fig. 8: Regression

\section{Conclusion}

In this work an analysis is done on smart meter data for shortrange ,distinct and cumulative dataset estimating the residential electricity consumption. Smart meter helps to get the energy data, which identifies demand response, abnormal load behaviors and fault diagnosis. The analytics performed will help stakeholders or consumers the energy consumption details and enough insights of the data. Energy demand forecasting has been broadly studied in the literature by examining the various mechanisms and techniques. Consumers preserve ownership and fine-grained control over their energy consumption data while enabling third party applications to examine the data in a secrecy preservative fashion. However demand keeps on growing exponentially which could lead for a large scale data their by, different forecasting technique as to be implemented in a Cost effective way for the future power consumption.

\section{References}

[1] DammindaAlahakoon, Member, IEEE, and Xinghuo Yu, Fellow, IEEE. IEEE transactions on industrial informatics, vol. 12, no. 1, February 2016 Smart Electricity Meter Data Intelligence for Future Energy Systems: A Survey Advanced Analytics for Harnessing the Power of Smart Meter Big Data

[2] Advanced meter infrastructure Available at:"http://www indiasmartgrid.org/Advanced-Metering-Infrastructure.php"

[3] A. JarrahNezhad, T. K. Wijaya, M. Vasirani, and K. Aberer, "SmartD:Smart Meter Data Analytics Dashboard," in the 5th ACM InternationalConference on Future Energy Systems (e-Energy'14), 2014.

[4] Tri KurniawanWijaya, MatteoVasirani, Samuel Humeau, Karl Aberer School of Computer and Communication Sciences EPFL, Switzerland Email: \{tri-kurniawan.wijaya, matteo.vasirani, samuel.humeau, karl.aberer\}@epfl.ch 2015 IEEE International Conference on Big Data Cluster-based Aggregate Forecasting for Residential Electricity Demand using Smart Meter Data

[5] T. K. Wijaya, M. Vasirani, and K. Aberer, "When bias matters: An economic assessment of demand response baselines for residential customers," IEEE Transactions on Smart Grid, vol. 5, no. 4, pp. 1755-1763, July 2014

[6] RaymanPreet Singh, S. Keshav, and Tim Brecht \{rmmathar, keshav,brecht\}@uwaterloo.ca School of Computer Science, University of Waterloo, 2015.

[7] A Cloud-Based Consumer-Centric Architecture for Energy Data Analytics. Waterloo, Ontario, Canada [2] Carlos alzate, mathieusinnibm research - irelandmulhuddart, dublin15, Ireland $\{$ carlos.alzate, mathsinn $\} @$ ie.ibm.com. 2013 IEEE 13th International Conference on Data Mining Improved electricity load forecasting via kernel spectral clustering of smart meters.
[8] Amr A. Munshi, Student Member, IEEE, and Yasser A. I Mohamed, Senior Member, IEEE Cloud-Based Visual Analytics for Smart Grids Big Data

[9] DRED data sets Available at - http://www.st.ewi.tudelft. $\mathrm{nl} / \sim$ akshay/dred

[10] S. Haben, J. Ward, D. V. Greetham, C.Singleton, and P. Grindrod, "A new error measure for forecasts of household-level, high resolution electrical energy consumption," International Journal of Forecasting, vol. 30, no. 2, pp. 246 - 256, 2014

[11] M. Chaouch, "Clustering-based improvement of nonparametric functional time series forecasting: Application to intra-day household-level load curves," IEEE Transactions on Smart Grid, vol. 5, no. 1, pp. 411-419, Jan 2014.

[12] T. K. Wijaya, "Pervasive Data Analytics for Sustainable Energy Systems,” Ph.D. dissertation, EPFL, Lausanne, 2015.

[13] Joseph F.Hair JR, William C.Black, Barry J.Babin, RolphE.Anderson" Multivariate Data Analysis (Book)", 2014,p. 30-200

[14] Math Works Documents Available at:https://in.mathworks. com/help/econ/arima.forecast.html

[15] Feed forward, Available at: http://in.mathworks.Com /help/nnet/ref/feedforwardne t.html

[16] Linear regression, Available at: http://reliawiki.org/index. php/Simple_Linear_Regres sion_Analysis 